\title{
Conversational Problem Solving
}

\section{Reviewed by Mohamed Omar}

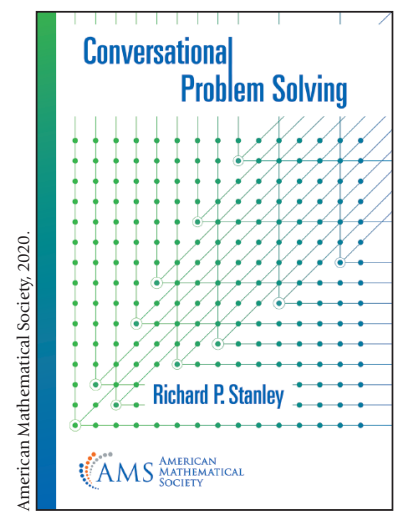

printed copies of past math competitions and the odd book at the library or bookstore kept me captivated for hours. I even remember bringing George E. Andrews' book Number Theory to my math classes, sitting in the back corner, and intensely reading the book in admitted confusion, trying to figure out how things would unfold before reading about them. Many books like this had exposition at a level I couldn't understand. They made me yearn for the feeling of being in a classroom discovering fun and interesting theorems or math facts with a group of similarly excited students, rather than struggling through the drudgery of lonesome reading. Now two decades later, Conversational Problem Solving by Richard P. Stanley, in many but not all respects, is that book I was seeking.

Conversational Problem Solving is a fun journey through mathematical problem solving and discovery. The book

Mohamed Omar is an associate professor of mathematics and Joseph B. Platt chair at Harvey Mudd College. His email address is omar@g . hmc. edu. Communicated by Notices Book Review Editor Stephan Ramon Garcia.

For permission to reprint this article, please contact: reprint-permission aams.org.

DOI: https://dx.doi.org/10.1090/noti 2341 features amusing characters in a Problem Solving camp led by Professor Mortimer Ignatius Blakeley, and takes the reader through the many days of the camp. The book is intended mostly for an undergraduate audience in the lens of challenging problem solving, akin to what one would encounter when participating in university-level math competitions (such as the Putnam Competition, Virginia Tech Regional Mathematics Competition, International Mathematics Competition, or the like). However, the book is also accessible to high school students who have extensive mathematical exposure and experience. Indeed, the content for most of the book was inspired by a course on mathematical problem solving taught by the author while at MIT. The book is written in a conversational tone where the viewpoint of the reader is as a student in the classroom sitting in the back corner observing the interactions between students and Professor Blakeley. A great excerpt that sets the tone is the following quote from page 37 :

Most of the students had seen this puzzle, but a few had not. After giving them a little time to think about it, Professor Blakeley said, "Here is a hint-the sequence is constant."

This hint seemed only to deepen the mystery for the students not already familiar with the problem, but then Fumei saw the light. "I got it!" she said.

The reader is led through the discovery process with Prof. Blakeley's class. Because of this, the manner in which the book is written quite naturally gives the reader the opportunity to pause and freely investigate. I think this is extremely important for students engaging with mathematical problem solving material. In this way, students not only learn interesting and new problem solving techniques, they actually experience the problem solving practice 
themselves. A lovely added bonus is that the book's layout lets students easily investigate whatever style of problem they desire. Each chapter has, at the forefront, a list of the problems that are discussed in the chapter, with the page number that each problem's discussion appears on. When I was a student, I knew I had a taste for a specific class of problems, so this would have made things very intriguing; not having to read the book linearly in order to engage fully.

Another aspect of this book I sincerely appreciated was how akin the experience of reading it actually was to experiences at an actual mathematics camp. In the summers of 2011 and 2012, I worked as a graduate student mentor and academic coordinator at the Canada/USA Mathcamp. This camp is attended annually by 120 extremely enthusiastic students who engage deeply with their mathematical curiosities during the entire 5-week period of the camp. I remember the conversations that would transpire at the camp and they are truly reflected in this book. A mathematical pattern emerges and it sparks deep curiosity. That curiosity leads to investigation, bouts of euphoria and bewilderment, conjectures that fail or come true, and illumination that bridges campers to other problems and mathematical gems. The book exemplifies this experience extremely accurately. Reading it, I felt myself being transported to the Mathcamp classrooms in a way I haven't felt in a very long time.

Finally, as to my overall impression, I think this book actually can serve as a fantastic source for professors interested in engaging ways to introduce combinatorics topics to students. Many of the examples and problems in the book are combinatorial in flavor (which makes sense given who the author is). The book is packed with combinatorial gems found in Enumerative Combinatorics: Volume I, written by the same author, but introduced in this book from a more playful perspective. I could see this being a great source for a course on combinatorics with a small class size, especially if the class was flipped.

Now to the content. The first chapter provides a gentle introduction to the tone of the book without getting into too much mathematics that is technically heavy. It starts off by asking for the minimum number of planar cuts required to cut a $3 \times 3 \times 3$ cube into $271 \times 1 \times 1$ cubes. A particular student then presents a solution that takes into consideration analysis after the first slice. The student is then followed up by another student who presents an elegant solution that considers the center cube and how it requires 6 separate cuts to remove it since it has 6 faces, then establishes a decomposition with 6 cuts. The problem and solution here are not what's inspiring, but rather the tone that is set by them. It gives the reader the hint, an accurate hint, that the book explores problems but asks for more than solutions. It asks for perspectives, especially ones that illuminate the heart of what the problem aims to convey. And this is definitely a running theme throughout the exposition.

The first chapter goes on to look at generalizations of this problem to more complicated shapes, and cake cutting results in general. It guides students in the class through exploring possible solutions to these generalizations, ending with a nice exposition on what is known and not known about general cake cutting problems. This is a beautiful bridge from experimentation on a seemingly simple problem, to the uncovering of very interesting and current mathematics. I personally love this type of exposition as it is something I do often in my classes, especially when teaching a standalone course on combinatorics.

The mathematics starts to ramp up in the second chapter, dedicated to many interesting properties of polynomials. It focuses quite a bit on matrix techniques for determining real-rootedness and log concavity, both of which are ubiquitously sought after in combinatorics. With the possible caveat of needing to introduce determinants, I think this chapter is particularly amenable to a high school student who is deeply interested in mathematics. Much of the material is a nice extension of familiar ideas from the high school curriculum but ramped up quite heavily, so these students would appreciate the results.

Chapter 3 titled "Base Mathematics" is filled with many quick enjoyable gems. A particular one that seems very surprising to the naked eye until one processes it is the relationship between the decimal expansion of the fraction 100/9899 and Fibonacci numbers. Here is a truncated expansion:

$$
100 / 9899=0.01010203050813213455 \ldots
$$

Up until the 20th digit after the decimal place, the digits taken a pair at a time are the first several numbers in the Fibonacci sequence. This seems startling at first until one processes that the generating series $F(x)$ for the Fibonacci sequence satisfies $\left(1-x-x^{2}\right) F(x)=1$, so the decimal expansion can be seen as evaluating $x F(x)$ at $x=1 / 100$. Moreover, we see that we can develop such a decimal, containing arbitrarily long prefixes of the Fibonacci sequence, by substituting arbitrarily high powers of 10 in for $x$. The beginning of this chapter focuses on interesting evaluations like this involving the Catalan numbers as well. These curiosities, in conjunction with the conversational nature of the book, reinforce its enjoyable and fun tone.

The remainder of the book touches on many topics, so I'd like to highlight things I especially appreciated. As a problem solving enthusiast and instructor of the Putnam training sessions at my own institution, some of these are the gems that I hope to share with my students.

A gem I particularly appreciated was the geometric probability problems at the end of the 7 th chapter. These problems are typically framed as having $n$ points uniformly distributed over some geometric object and asking what the probability that some geometric phenomenon among them occurs. For example, one problem asks for the probability that $n$ points on the circumference of a circle lie on the same arc subtended by a fixed angle. These problems, 
as they get more complicated, tend to evade students. The book gives an example of such a problem from the 1992 Putnam, where only sixteen contestants successfully earned 8 or more out of 10 points, nobody earning between $2-7$ inclusive (out of thousands of contestants). After reading the discussion of this problem, a solution reveals itself quite nicely.

On top of the gems I am eager to share with my students, as a combinatorics researcher, I sincerely appreciated the nostalgia I experienced reading the book. So much of the content covers combinatorics I learned as an undergraduate and graduate student. In light of this, I highly recommend the book as a fun introduction to topics in combinatorics without the formalism of standard combinatorics books. This is especially true if one is teaching a combinatorics course that has a generating function emphasis. There are enough problems and curiosities in the book to make it a great supplement for such a course.

Finally, the one thing I found less appealing about this book was the overall tone. I think back to myself as a younger student and the impressions that tone made on me in feeling accepted in the mathematics community. Despite me having a strong background and doing reasonably well, the culture and language used by aspiring and active mathematicians tended to be condescending and diminutive. That made me uncomfortable to share my progress while solving problems. If I picked up Conversational Problem Solving late in high school or early in my undergraduate years, I think it might have deterred me from engaging in extracurricular mathematics altogether. In particular, as enthusiastic as Professor Blakeley is, there is an underlying condescending tone laced with declaring problems as "easy," "trivial," or "obvious." Especially unnerving is declaring that students in the class should be able to solve particular problems. I think this tone misses the mark on possibly attracting many students who would want to happily engage with the material. I found this to especially be the case in Chapter 9, titled "Amanda." In this chapter, an enthusiastic ten or eleven-year-old enters the class and is able to have deep insight to the heart of a problem that other students in the class don't see. She proceeds with the quote "It seems so easy that I might have made a mistake" when solving another problem. I think the intention here is to highlight that there are nice, simple perspectives that illuminate the heart of a problem and avoid the drudgery of long computations and analyses. But I think the tone here again is not encouraging for an aspiring problem solver.

Overall, Conversational Problem Solving is a delightful book packed with many interesting problem solving strategies, beautiful combinatorial theorems and perspectives, fun quirks, and lessons that would complement an undergraduate math education quite nicely. Reading it filled me with inspiration and ideas for my own problem solving practice, leading to a list of interesting problems and ideas to share with students in the future. It also took me down combinatorics memory lane in a way I truly didn't expect and truly appreciated.

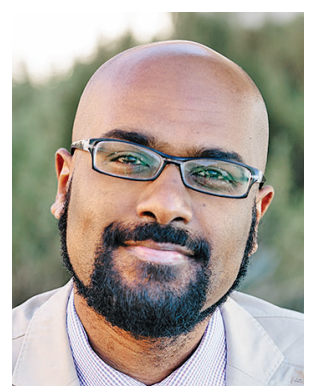

Mohamed Omar

\section{Credits}

Author photo is courtesy of Mohamed Omar.
When wes the last time pou wisited the

AMS Bookstore?

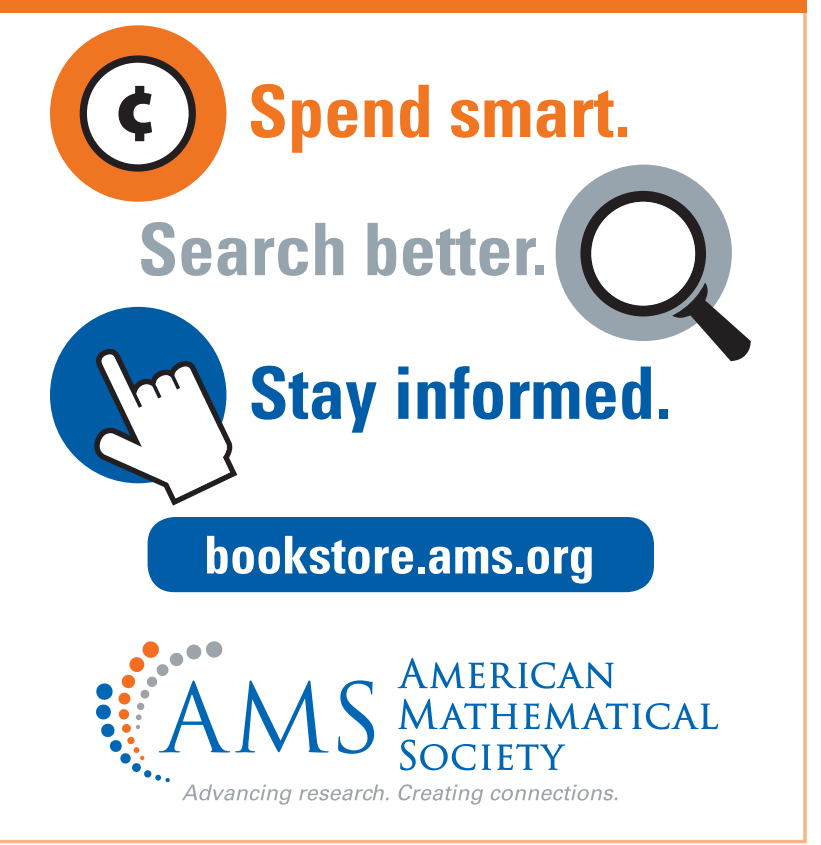

